\title{
THE DISTRIBUTION OF THE INFLATION IN TURKEY
}

\author{
Res.Asist.Dr. Nazif ÇATIK, Ege University, Department of Economics, Izmir-Turkey. \\ Prof. Dr. A. Özlem ÖNDER, Ege University, Department of Economics, Izmir-Turkey.
}

\begin{abstract}
This paper investigates distribution of inflation items using various measures of skewness and kurtosis for Turkey covering the period 1996-2007. Considering sensitivity of traditional distribution measures to outlying observations robust skewness and kurtosis are also computed as a novelty. Analysis results mainly reveal that inflation components are right skewed and fat tailed as documented by the previous studies. However due to possible effects of the outliers traditional measures, in particular skewness, are lagging behind the robust measures in identifying underlying dispersion. Therefore one can say that weighted mean inflation used to measure general price changes is not trustworthy and a biased estimator of central location. Our results further suggest that core measures based on constant and symmetric trimming applied by the previous studies for Turkey is somewhat deficient since skewness of the data is ignored in the estimation process. Therefore, to obtain more reliable information in monitoring general price movements we suggest use of asymmetric trimmed means estimators which allows trimming different percentages from each tail of the distribution.
\end{abstract}

Key Words: Inflation, Robust Skewness and Kurtosis

\section{INTRODUCTION}

It is often argued that inflation data consisting of expenditure items does not follow the characteristics of normal distribution in most of the countries. Studies related to measurement and distribution of inflation conducted for different countries have reached the stylized fact that cross-sectional items of price changes are skewed to right and also have excess kurtosis (see for example Bryan and Pike (1991Kearns (1998), Cecchetti (1997), Roger (1997)). 
In those studies traditional skewness and kurtosis measures based on third and fourth moments of inflation components have been used to explain how the data is dispersed around the sample mean (see for example Berkmen (2002), Kucuk and Tuger (2004), Atuk and Ozmen (2009) for Turkey). However sample mean itself has zero breakdown point, this makes those dispersion measures are largely affected by outlying observations. In other words only one outlying observation is enough to keep the sample mean away from "true" centre of the data, which is already known as masking effect in the statistics literature. In this case headline inflation based on the weighted average of items may not be good representative of the general price movements in the economy.

Given the fact that measures of skewness and kurtosis are not robust, this paper is aimed to evaluate distribution of inflation using both robust and traditional measures of dispersion. To our best knowledge this study is first attempt to measure distribution of inflation components using robust measures for Turkey. By doing this we would like to assess reliability of weighted mean inflation as an indicator of general price movements. Even though inflation components are found as right skewed and fat tailed in Turkey for the period 1996-2007, our dispersion measures based on different time spans mainly reveal that traditional measures, in particular skewness may fail capturing "true" dispersion of the data. Given the highly volatile and skewed characteristics of the price changes we concluded that symmetric trimmed means applied by previous studies may not provide efficient estimation of central location, therefore we need to use estimators where the skewed behavior of the data is taken into consideration.

This paper is organized as follows. In the next section we will give brief description of skewness and kurtosis measures used in the paper. Skewness and kurtosis coefficients have been computed and evaluated in the third section. Finally in the last section some conclusions are put forward.

\section{MEASUREMENT OF ROBUST SKEWNESS AND KURTOSIS}

\subsection{Data and Traditional Measures of Dispersion}

This study is based on monthly Turkish Harmonised Indices of Consumer Prices (HICP) data obtained from Eurostat Database ${ }^{1}$. For each month we have 75 twodigit level disaggregated prices of HICP and their yearly weights covering the period 1996:2-2007:5. We also used seasonally adjusted data to make sure seasonal components have significant effects on the distribution of inflation. Our investigation period is determined by the availability of the data.

\footnotetext{
${ }^{1}$ Eurostat web site is http://epp.eurostat.ec.europa.eu/portal/page/portal/eurostat/home.
} 
In the calculation of dispersion measures four time spans $k$ from month on month to year on year $k=1,3,6,12$ have been considered. Using those components and weights it's possible to compute headline inflation in terms of weighted average of items as follows:

$$
\pi=\sum_{i=1}^{N} w_{i} \pi_{i}
$$

Under the assumption of cross-sectional monthly inflation rates $\pi_{i}$ are independently and identically distributed with a cumulative distribution function $F$, conventional skewness and kurtosis for $\pi_{\mathrm{i}}$ can be written as:

$$
S K=\frac{\sum_{i=1}^{N} w_{i}\left(\pi_{i}-\pi\right)^{3}}{\sigma^{3}} \quad K=\frac{\sum_{i=1}^{N} w_{i}\left(\pi_{i}-\pi\right)^{4}}{\sigma^{4}}
$$

Where standard deviation of inflation components is $\sigma=\sqrt{\sum_{i=1}^{N} w_{i}\left(\pi_{i}-\pi\right)^{2}}$. It's well known that in normally distributed data skewness and kurtosis should be equal to 0 and 3 respectively.

Using third and fourth moments of cross-sectional price changes in (2) Jargue-Bera statistics can be computed by

$J B=N\left(\frac{S K^{2}}{6}-\frac{(K-3)^{2}}{24}\right)$

However conventional skewness and kurtosis coefficients described in (2) can not be taken as robust measures of dispersion, since they are defined in terms of deviation from the sample averages already known as having zero breakdown point $^{2}$. In other words only one observation can make these measures inconsistent estimators of central tendency. In order to overcome this problem several robust

2 The breakdown point gives the limiting fraction of bad outliers the estimator can cope with. See Huber (1981) and Rousseeuw and Leroy (1987) for detailed information. 
skewness and kurtosis measures who can cope with data containing a certain percentage of outliers have been proposed in the literature.

Existence of outlying observations is much more evident in the measurement of inflation, since inflation data contain many items subject to seasonal change, i.e. vegetables and seafood and also managed prices by the government, such as electricity and fuel prices and this makes harder to find true dispersion of the data with conventional measures especially based on sample averages. Therefore use of robust location and dispersion measures resistant to certain amount of outliers has of crucial importance in determining underlying characteristics of price changes for Turkey.

\subsection{Robust Measures of Kurtosis}

A robust measure of kurtosis is proposed by Crow and Siddiqui (1967):

$$
K_{2}=\frac{F^{-1}(1-\alpha)+F^{-1}(\alpha)}{F^{-1}(1-\beta)-F^{-1}(\beta)}-2.91
$$

$\alpha$ and $\beta$ values in the inverse cumulative distribution function can take any value between 0 and 1 . In this study $\alpha$ and $\beta$ are determined as 0.025 and 0.25 respectively as in Crow and Siddiqui (1967). Using those reference values, quartiles can be easily obtained as $\mathrm{F}^{-1}(0.975)=-\mathrm{F}^{-1}(0.025)=1.96$ and $\mathrm{F}^{-1}(0.75)=\mathrm{F}^{-1}(0.25)=-0.68$ for normal distribution and normal value for Crow and Siddiqui kurtosis coefficient is equal to 2.91 under normal distribution. Considering weakness of traditional skewness measure in the presence of outliers Moors (1988) proposed the following robust measure:

$$
K_{1}=\frac{\left(E_{7}-E_{5}\right)+\left(E_{3}-E_{1}\right)}{E_{6}-E_{2}}-1.23
$$

Where $E_{i}$ is the first octile represents $1 / 8$ portion of the data, and alternatively expressed as $E^{i}=F^{-1}(i / 8)$ for $\mathrm{i}=1,2, \ldots 7$. For a standard normal distribution $\mathrm{N}(0,1)$ it is straightforward to calculate that $\mathrm{E}_{1}=-\mathrm{E}_{7}=-1.15, \mathrm{E}_{2}=-\mathrm{E}_{6}=-0.68$, $E_{3}=E_{5}=-0.32$ and $E_{4}=0$ and therefore normal value for Moors kurtosis is equal to1.23. 


\subsection{Robust Measures of Skewness}

In order to compute the robust measures of location and dispersion several methods are considered. Bowley (1920) proposed the following measure called as quantile skewness in the literature:

$$
S K_{1}=\frac{\left(Q_{3}-Q_{2}\right)-\left(Q_{2}-Q_{1}\right)}{Q_{3}-Q_{1}}=\frac{Q_{3}+Q_{1}-2 Q_{2}}{Q_{3}-Q_{1}}
$$

Qi is the ith quartile of cross-sectional components price changes, in the above formula. In case of standard normal distribution Bowley skewness is should be zero. Since this measure is standardized by the difference between third and first quartile , it lies within the boundaries of -1 and +1 . Negative values indicates left skewness whereas positive coefficients represents right skewness.

Another robust measure of skewness is suggested by Groeneveld and Meeden (1984) based on the following formula:

$$
S K_{2}=\frac{\pi-Q_{2}}{E\left|\pi-Q_{2}\right|}
$$

If the denominator of Groeneveld and Meeden skewness replaced by standard deviation, Pearson coefficient another robust measure of skewness can be obtained as follows:

$$
S K_{3}=\frac{\pi-Q_{2}}{\sigma}
$$

\section{APPLICATION OF STATISTICS TO THE TURKISH DATA}

In this section we compute traditional and robust measures of skewness and kurtosis for the Turkish HICP data described in the previous section.

First we have computed traditional measures of skewness and kurtosis for each individual cross-section. Even though average values of traditional measures record fat tailed and right skewed distribution of price changes consistent with the previous findings, cross-sectional values depicted in Figures 1 and 2 reports existence of left skewness in some periods due to possible effects of outlying 
observations (see Table 1). It is also remarkable that use of both seasonal and nonseasonal data have produced the qualitatively the same results (see Figure 1 and 2). This finding confirms the fact that seasonal adjustment itself may not be sufficient ensuring normality; hence we should take different methods to remove outlying observations from the data.

Next we use robust measures to estimate dispersion of the data. Average values are displayed in Table 1 confirm the previous findings on the distribution of price changes. Robust measures imply large positive skewness, i.e. they are close to one nearly in all time spans. They have even recorded positive values at all crosssections where traditional skewness supports the opposite. Similarly robust kurtosis values are also found to be very high, but have gradually decreasing tendency with the increase in time span. These results are also in line with the findings obtained from seasonally adjusted data.

Jarque-Bera and Mean percentile support the findings obtained from robust measures. Jarque-Bera statistic indicates that cross-sectional price changes are not distributed normally during this period. Average value of that measure is decreasing function of time interval $k$. Mean percentile which illustrates fractional location of the average inflation within the items shows extremely volatile and asymmetric behavior over the period, on average it's usually above the median which corresponds to $50^{\text {th }}$ percentile of the data (see Figure 3). This finding gives justification for the use of core inflation measures based on trimming extreme observations from each tail of the distribution ${ }^{3}$. It also suggests that constant and symmetric trimming would not be enough ensuring normality and therefore asymmetric trimming should be applied to capture general trend of the data ${ }^{4}$.

In general, robust measures computed here more strongly supportive of the stylized fact on the distribution of price changes than previously obtained from conventional skewness and kurtosis, those measures further verify that right skewness, high

\footnotetext{
3 The term of Core inflation is first introduced by Eckstein (1981). In that study core inflation is defined as the trend increase of the cost of the factors of production and disturbances have only transitory impacts on inflation usually associated with supply shocks

4 Trimmed means method is first used by Bryan and Pike (1991) and Bryan and Cecchetti (1993) in the estimation of core inflation. Considering skewed properties of cross-sectional price changes Kearns (1998), Roger (1997), Bryan and Cecchetti (2000) and Margues and Mota (2000) have modified this method. They suggest centering the data different from the 50th percentile to allow for asymmetry. Another method is proposed by Aucremanne (2000) where optimal trimming percentage is determined as the lowest percentage for which the hypothesis of normality is not rejected by Jarque-Bera statistic. Berkmen (2002), Atuk and Ozmen (2009) have estimated core inflation using trimmed means approach for Turkey. Although distribution of price changes have been found right skewed and leptokurtic only symmetric trimming case is considered by those studies.
} 
kurtosis and non-normality are fundamental characteristics of the Turkish inflation data.

\section{CONCLUSIONS}

In this paper we aimed to evaluate distribution of inflation in Turkey for the period 1996:2-2007:5. Considering sensitivity of traditional measures to outlying observations robust skewness and kurtosis are also applied to the Turkish HICP data for the first time. Inflation components are found to be right skewed and fat tailed as documented by the previous studies. However because of the possible effects of outliers, traditional measures, in particular skewness are lagging behind the robust measures in determining true dispersion of the data. We also compute those statistics using seasonally adjusted data but results are qualitatively the same with the one obtained from the original. In general robust skewness and kurtosis measures perform better than the conventional measures. Our results mainly verify the fact that weighted mean inflation used to measure general price changes in overall economy is not robust and a biased estimator of central location. Our results further suggest that constant and symmetric trimming applied by the previous studies for Turkey is somewhat deficient since skewness of the data is ignored in the estimation process. Therefore we suggest use of estimators like asymmetric trimmed means where the skewed behavior of the data is taken into consideration and this may provide more reliable information in monitoring general price movements than the weighted mean inflation itself. 
Table 1. Kurtosis and Skewness Measures of Turkey's HICP Inflation

\begin{tabular}{|l|c|c|c|c|c|c|c|c|}
\hline & \multicolumn{3}{|c|}{ Seasonally Unadjusted } & \multicolumn{3}{c|}{ Seasonally Adjusted } \\
\hline & $\mathbf{k}=1$ & $\mathbf{k}=3$ & $\mathbf{k}=6$ & $\mathbf{k}=12$ & $\mathbf{k}=1$ & $\mathbf{k}=3$ & $\mathbf{k}=6$ & $\mathbf{k}=12$ \\
\hline Kurtosis Measures & & & & & & & & \\
\hline Kurtosis (Normal) & 13.095 & 10.063 & 7.589 & 5.227 & 11.276 & 7.682 & 5.302 & 1.079 \\
\hline Moors Kurtosis & 0.402 & 0.411 & 0.228 & 0.090 & 0.414 & 0.411 & 0.170 & 0.079 \\
\hline Crow and Siddiqui & 0.437 & 0.452 & 0.578 & 1.341 & 0.338 & 0.386 & 0.723 & 1.328 \\
\hline Skewness Measures & & & & & & & & \\
\hline Skewness (Normal) & 1.568 & 1.329 & 1.192 & 1.084 & 1.456 & 1.095 & 0.927 & 1.079 \\
\hline Bowley & 0.176 & 0.138 & 0.109 & 0.073 & 0.044 & 0.054 & 0.065 & 0.075 \\
\hline Pearson & 0.158 & 0.132 & 0.104 & 0.090 & 0.079 & 0.078 & 0.077 & 0.091 \\
\hline Gronweld and Meeden & 0.286 & 0.225 & 0.164 & 0.132 & 0.147 & 0.128 & 0.114 & 0.134 \\
\hline
\end{tabular}


Figure 1. Kurtosis Measures for Turkish HICP Data
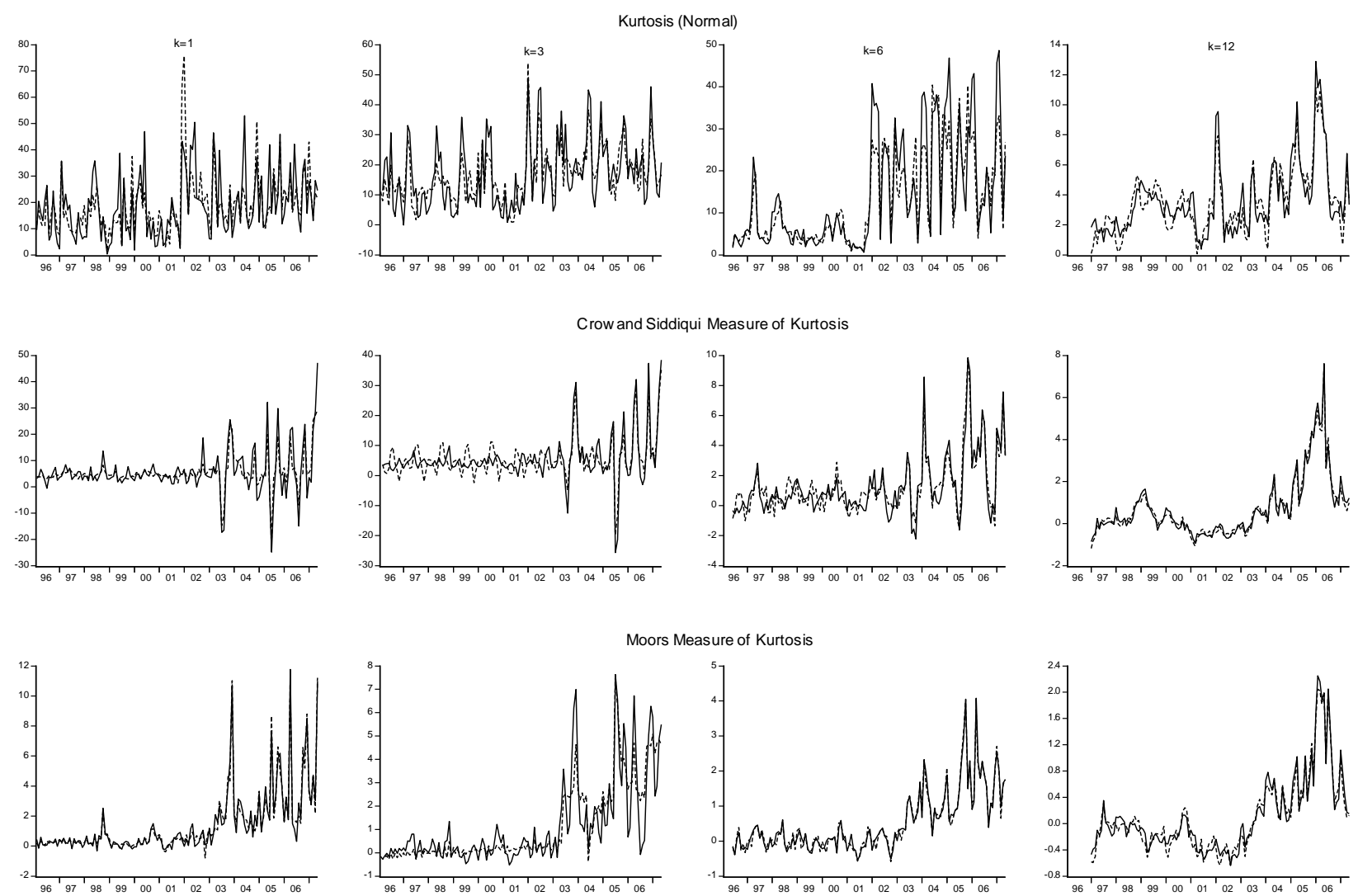

Note: Measures of kurtosis obtained from seasonally adjusted items are represented by dashed lines. 
Figure 2. Skewness Measures for Turkish HICP Data
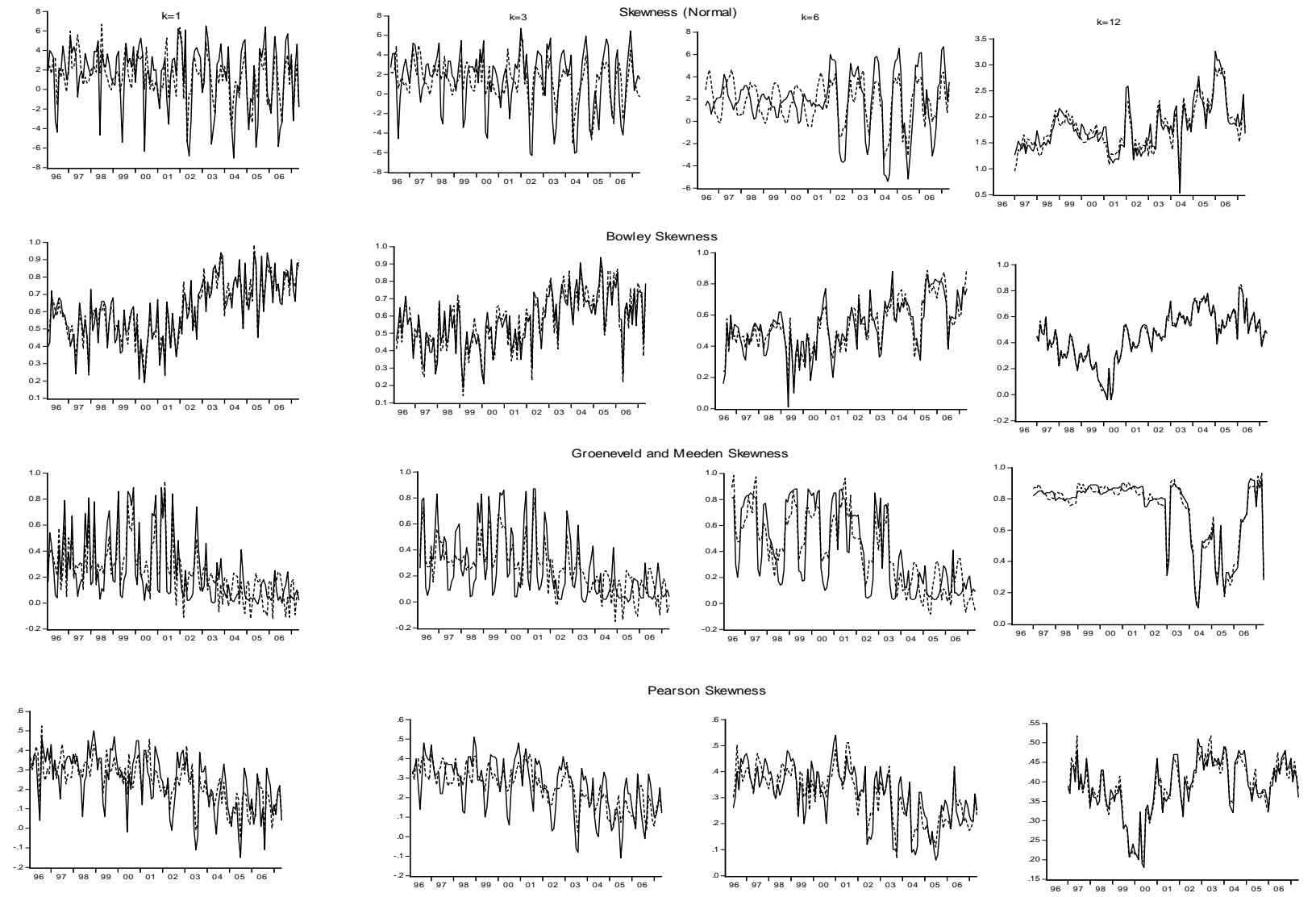

Note: Measures of skewness obtained from seasonally adjusted items are represented by dashed lines. 
Figure 3. Normality of the Data: Mean Percentiles and Jarque-Bera Statistics
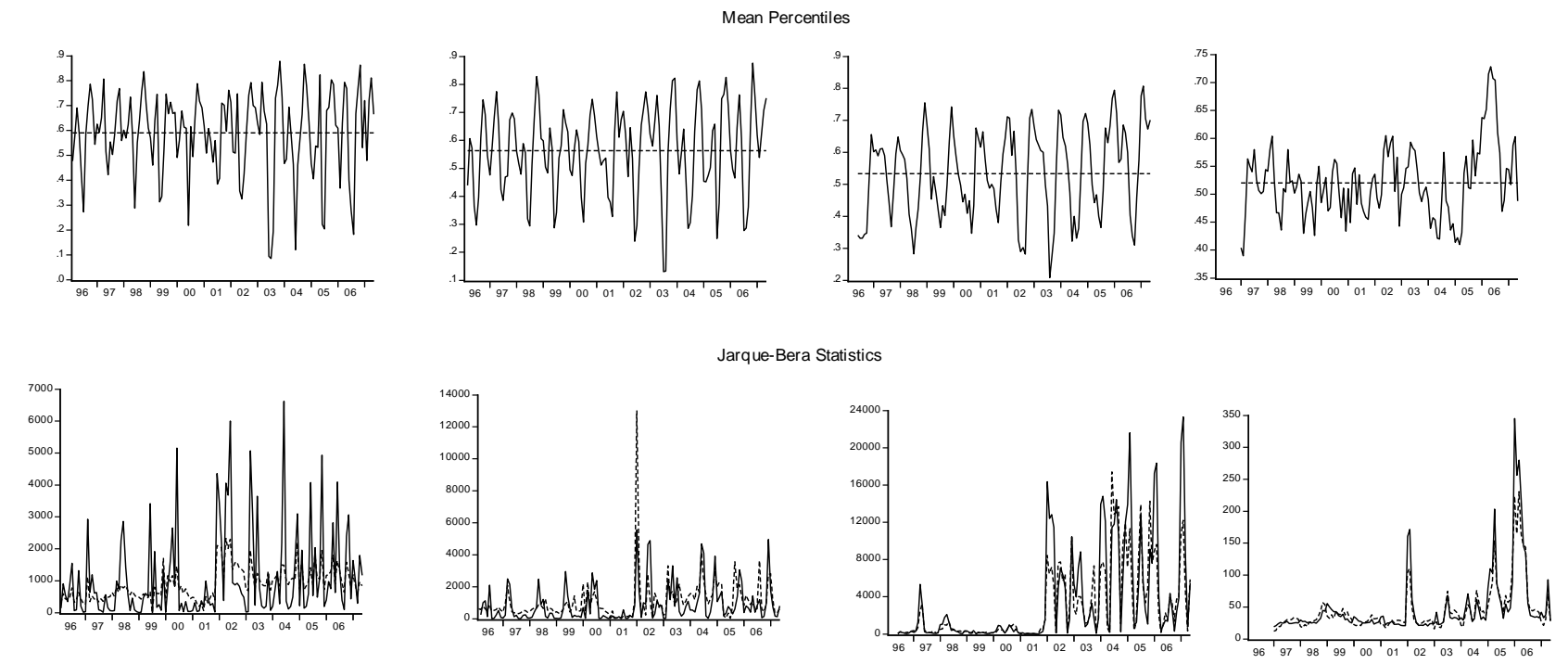

Notes: Straight dashed lines show average mean percentile in mean percentile figures. Seasonally adjusted Jarque-Bera statistics are given by dashed lines. 


\section{References}

Atuk O. and Özmen M. U. (2009), "Design and evaluation of core inflation measures for Turkey," IFC Working Papers, No 3.

Aucremanne, Luc (2000) "The Use of Robust Estimators as Measures of Core Inflation," National Bank of Belgium Working Paper, No. 2.

Berkmen P. (2002), "Measuring Core Inflation for Turkey Trimmed Means Approach," Central Bank Review, 2002, 2 (2): 1-18.

Bowley, A.L. (1920), Elements of Statistics, New York: Charles Scribner's Sons.

Bryan, M. F. and S. G. Cecchetti (1993), "The Consumer Price Index as a Measure of Inflation," Economic Review of the Federal Reserve Bank of Cleveland, Vol. 29/4, pp. $15-24$.

Bryan, Michael F., and Cecchetti, S. G. (2000), "A Note on the Efficient Estimation of Inflation in Brazil," Banco Central do Brasil Working Paper, No. 11.

Bryan, M.F. and Pike, C.J. (1991), "Median Price Changes: An alternative approach to measuring current monetary inflation," Federal Reserve Bank of Cleveland Economic Commentary, December, pp. 1-4.

Cecchetti, S.G., (1997), " Measuring short-run inflation for central bankers," Review of the Federal Reserve Bank of St. Louis, 79, number 3, 143-155.

Crow, E.L. and Siddiqui, M.M. (1967), "Robust Estimation of Location," Journal of the American Statistical Association, 62, 353-389.

Groeneveld, R.A. and Meeden, G. (1984), "Measuring Skewness and Kurtosis," The Statistician, 33, 391-399.

Huber, Peter. J. (1981), Robust Statistics, New York: John Wiley and Sons.

Kucuk, H and Tuger, B. (2004), "Relative Price Variability: The Case of Turkey 1994-2002", Central Bank of Turkey Research Department Working Paper, No:04/02.

Kearns, J. (1998), "The distribution and Measurement of Inflation", Reserve Bank of Australia Discussion Paper, no. 9810. 
Marques, C. R. and Mota (2000) "Using the asymmetric trimmed mean as a core inflation indicator," Banco de Portugal Working Papers, No. 6 (October).

Moors, J. J. A. (1988), "A Quantile Alternative for Kurtosis," The Statistician, 37, 2532.

Roger, S., 1997. A Robust Measure of Core Inflation in New Zealand, 1949-96. Reserve Bank of New Zealand.

Rousseeuw, P. J. ve Leroy, A. M. (1987), Robust Regression and Outlier Detection, New York: John Wiley and Sons. 